\title{
PROCESSO DE DELIBERAÇÃO MORAL DOS ENFERMEIROS DE COMPETÊNCIA GERENCIAL E FISCALIZATÓRIA DOS CONSELHOS DE ENFERMAGEM
}

Luciana Ramos Silveiraㄹ, Flávia Regina Souza Ramos ${ }^{1}$, Dulcinéia Chizoni Schneider¹, Maria Isabel Saracibar Razquin², Laura Cavalcanti Farias Brehmer ${ }^{1}$

Objetivo: descrever o processo e os valores implicados na deliberação moral dos profissionais de competência gerencial e fiscais, considerando suas atribuições e os problemas éticos encontrados. Metodologia: estudo descritivo e exploratório, abordagem qualitativa, realizado durante o período de novembro 2013 a novembro de 2014, cujas informações foram coletadas por meio de questionário. Participaram da pesquisa 28 profissionais de competência gerencial e 113 profissionais de competência fiscalizatória dos departamentos de fiscalização do exercício profissional de enfermagem brasileira. Os dados foram organizados e analisados segundo a técnica de análise textual discursiva. Resultados: o itinerário proposto pelos participantes revela certa maturidade e conhecimento, constatado através das etapas eleitas. Conclusão: o estudo apontou que os trâmites do processo são fortemente vinculados a aspectos burocráticos e organizacionais. Convém ressaltar que os valores implicados no processo estão relacionados às dimensões ética, legal, subjetiva e profissional.

Descritores: Regulação e Fiscalização em Saúde; Enfermagem; Deliberação.

\section{MORAL DELIBERATION PROCESS OF MANAGEMENT AND SUPERVISORY COMPETENCIES OF THE NURSING} COUNCILS IN BRAZIL

Objective: describe the process and values involved in the moral deliberation of professionals with managerial and fiscal competencies, considering their responsibilities and ethical problems encountered. Methodology: Descriptive and exploratory study, qualitative approach, conducted from November 2013 to November 2014 and whose information was collected through questionnaire. There was a participation of 28 management professionals and 113 supervisory professionals from the professional inspection departments of the Brazilian nursing. Data were organized and analyzed according to the technique of discursive and textual analysis. Results: the itinerary proposed by the participants revealed certain maturity and knowledge, which was verified through the elected steps. Conclusion: the study pointed out that the process formalities are strongly linked to bureaucratic and organizational aspects. It is worth mentioning that the values involved in the process are related to ethical, legal, subjective and professional dimensions.

Descriptors: Regulation and Supervision in Health; Nursing; Deliberation.

\section{PROCESO DE DELIBERACIÓN MORAL DE LOS PROFESIONALES CON COMPETENCIA GERENCIAL Y FISCALIZADORA DE LOS} CONSEJOS DE ENFERMERÍA DEL BRASIL

Objetivo: describir el proceso y los valores implicados en la deliberación moral de los profesionales de competencia gerencial y fiscal, considerando sus atribuciones y los problemas éticos encontrados. Metodología: Estudio descriptivo y exploratorio, enfoque cualitativo, realizado durante el período de Noviembre del 2013 hasta Noviembre del 2014 y cuyas informaciones fueron obtenidas por medio de cuestionario. Participaron de la investigación 28 profesionales de competencia gerencial y 113 profesionales de competencia fiscalizadora de los departamentos de fiscalización del ejercicio profesional de la enfermería brasileña. Los datos fueron organizados y analizados según la técnica del análisis textual discursivo. Resultados: el itinerario propuesto por los participantes revela cierta madurez y conocimiento, lo que fue constatado a través de las etapas elegidas. Conclusión: el estudio determinó que los trámites del proceso están fuertemente vinculados a los aspectos burocráticos y organizacionales. Vale resaltar que los valores implicados en el proceso están relacionados con las dimensiones éticas, legales, subjetivas y profesionales.

Descriptores: Regulación y Fiscalización en Salud; Enfermería; Deliberación.

Universidade Federal de Santa Catarina-UFSC,Brasil.

Universidad de Navarra,Pamplona,Espanha.

Autora correspondente: Luciana Ramos Silveira. E-mail:Luramos76@yahoo.com

22 I Enferm Foco [Internet]. 2019:10(3): 22-27. 


\section{INTRODUÇÃO}

O foco deste estudo é a deliberação de problemas éticos no âmbito dos Conselhos Regionais de Enfermagem (Corens) brasileiros, entendendo a deliberação moral como um método que visa à análise dos problemas em toda a sua complexidade permitindo eleger cursos de ação possiveis para sua resolução, não como pura equação matemática, mas por uma análise cuidadosa e reflexiva sobre os eventos e circunstâncias envolvidas ${ }^{(1)}$.

Os problemas éticos, de forma geral, são questões de difícil condução, podem ser influenciados por valores, crenças, relações profissionais, entre outros aspectos, como aqueles inerentes às relações de poder e autoridade. Portanto, é necessário ter cautela e buscar a solução que seja mais prudente(2).

Mais especificamente no contexto deste trabalho, levam-se em consideração as etapas da deliberação utilizadas pelos enfermeiros de competência gerencial e de competência fiscalizatória dos Conselhos Profissionais de Enfermagem, quando esses analisam os problemas éticos vivenciados no seu cotidiano de trabalho. Vinculados aos Corens por meio do Sistema de Fiscalização do Exercício Profissional que, no caso da Enfermagem é regido pela Resolução Cofen no 374/2011 (3), esses profissionais estabelecem uma ligação mais próxima com o cotidiano laboral dos profissionais de enfermagem no intuito de manter os preceitos éticos e legais da profissão.

De acordo com a literatura, muitos são os problemas que emergem do exercício da enfermagem, incluindo profissionais não habilitados, em situação irregular, inseridos em condições de trabalho preocupantes, sobrecarregados devido a recursos humanos insuficientes, entre outros ${ }^{(4-5)}$. Considerando a relevância do trabalho realizado pelos enfermeiros gerentes e fiscais para a segurança da sociedade e a valorização da qualidade do cuidado da enfermagem brasileira, torna-se objeto de reflexão o processo de deliberação e os valores identificados como prudentes durante seu processo de trabalho.

Nas últimas duas décadas vários autores têm desenvolvido métodos para discutir e tentar solucionar conflitos que emergem da prática laboral, estudando processos éticos de enfermagem Schneider e Ramos ${ }^{(6)}$ chegaram a um método de deliberação moral em muito similar ao proposto por Gracia para a bioética clínica ${ }^{(1)}$, demonstrando que, independente do referencial teórico, o processo de deliberação é capaz de conectar todos os elementos necessários para uma visão mais abrangente das experiências ou problemas na prática profissional.

Assumindo tal pressuposto, o presente estudo teve como objetivo descrever o processo e os valores implicados na deliberação moral dos profissionais de competência gerencial e fiscais dos Corens brasileiros, considerando suas atribuições e os problemas éticos enfrentados. Trata-se de um dos objetivos da tese de doutorado intitulada "A fiscalização do exercício profissional de enfermagem no Brasil: desafios éticos", vinculada ao Programa de Pós-graduação em Enfermagem da Universidade Federal de Santa Catarina (UFSC), apoiada pelo CNPq.

\section{METODOLOGIA}

\section{Tipo de estudo}

Estudo descritivo exploratório, de natureza qualitativa.

\section{Participantes da pesquisa}

Optou-se por uma amostra intencional e não probabilistica, buscando alcançar representatividade de todos os conselhos. Foi possivel obter a participação de enfermeiros fiscais de todos os conselhos (total de profissionais 113) e de enfermeiros gerentes de 22 conselhos (total de profissionais 28). Para que a amostra fosse a mais universalizada possivel, não foram adotados quaisquer critérios de exclusão.

\section{Local do estudo}

O estudo desenvolveu-se nos Corens brasileiros. O Brasil é uma federação composta por 26 Estados e um Distrito Federal, todos apresentam uma sede de conselho de enfermagem, totalizando 27 conselhos. Os conselhos contam com um total de 52 enfermeiros de competência gerencial (gerentes) e 319 profissionais de competência fiscalizatória (fiscais), considerados como a população total desta pesquisa.

\section{Coleta dos dados}

Para a coleta de dados, utilizou-se um questionário construido pelas próprias autoras. $\mathrm{O}$ instrumento foi desenvolvido a partir de perguntas abertas e fechadas totalizando nove questões. O presente recorte contemplou uma questão aberta, no qual, abordou as etapas envolvidas no processo de tomada de decisão no enfrentamento de problemas éticos, considerando suas atribuições e os valores implicados.

Os dados foram coletados no período de novembro 2013 a novembro de 2014. Este momento envolveu três etapas: A primeira etapa da coleta foi presencial, após aprovação do Cofen, durante o 6o Seminário Nacional de Fiscalização do Exercício Profissional de Enfermagem com distribuição dos instrumentos impressos. A segunda etapa se deu através do envio do questionário por meio de um link eletrônico. $\mathrm{Na}$ terceira etapa optou-se pelo envio dos instrumentos por 
correio, endereçados, para os locais de trabalho. A segunda e terceira etapa foram estratégias para captar os participantes que não foram ao seminário e mobilizar os que optaram em não responder naquele momento. A mesma técnica foi utilizada para os enfermeiros gerentes e enfermeiros fiscais. Destaca-se que para todas as etapas foi solicitada a anuência dos participantes por meio do Termo de Consentimento Livre e Esclarecido (TCLE).

\section{Procedimentos de análise dos dados}

A análise e o tratamento dos dados foram orientados pelo método de Análise Textual Discursiva. Sua operacionalização envolveu três etapas: 1으 Desmontagem dos textos: examinaram-se as respostas dos participantes através de um exame detalhado e fragmentado; 2ㅇ Estabelecimento de relações: agruparam-se os elementos unitários em temas comuns, gerando as categorias: - Etapas da deliberação moral na perspectiva dos enfermeiros de competência gerencial e fiscalizatória dos Conselhos Profissionais de Enfermagem do Brasil; e, - Valores implicados no processo de deliberação moral.
3ㅇ Captação do novo emergente: concepção de uma renovada compreensão do todo.

\section{Procedimentos éticos}

O desenvolvimento do estudo seguiu os preceitos éticos disciplinados pela Resolução do Conselho Nacional de Saúde (CNS) 466/2012. A aprovação ética foi obtida pelo Comitê de Ética da Universidade Federal de Santa Catarina, mediante parecer no 372163. Foi garantido o anonimato e o sigilo aos participantes, adotando-se as denominações profissionais de competência gerencial e profissionais de competência fiscalizatória. Todos os envolvidos no estudo registraram a sua anuência por meio do Termo de Consentimento Livre e Esclarecido.

\section{RESULTADOS}

Considerando as suas atribuições e o enfrentamento de problemas éticos, os participantes elegeram sete etapas necessárias para tomar uma decisão. Assim, propõese neste estudo, a apresentação estrutural deste itinerário sistematizado de deliberação (Figural).

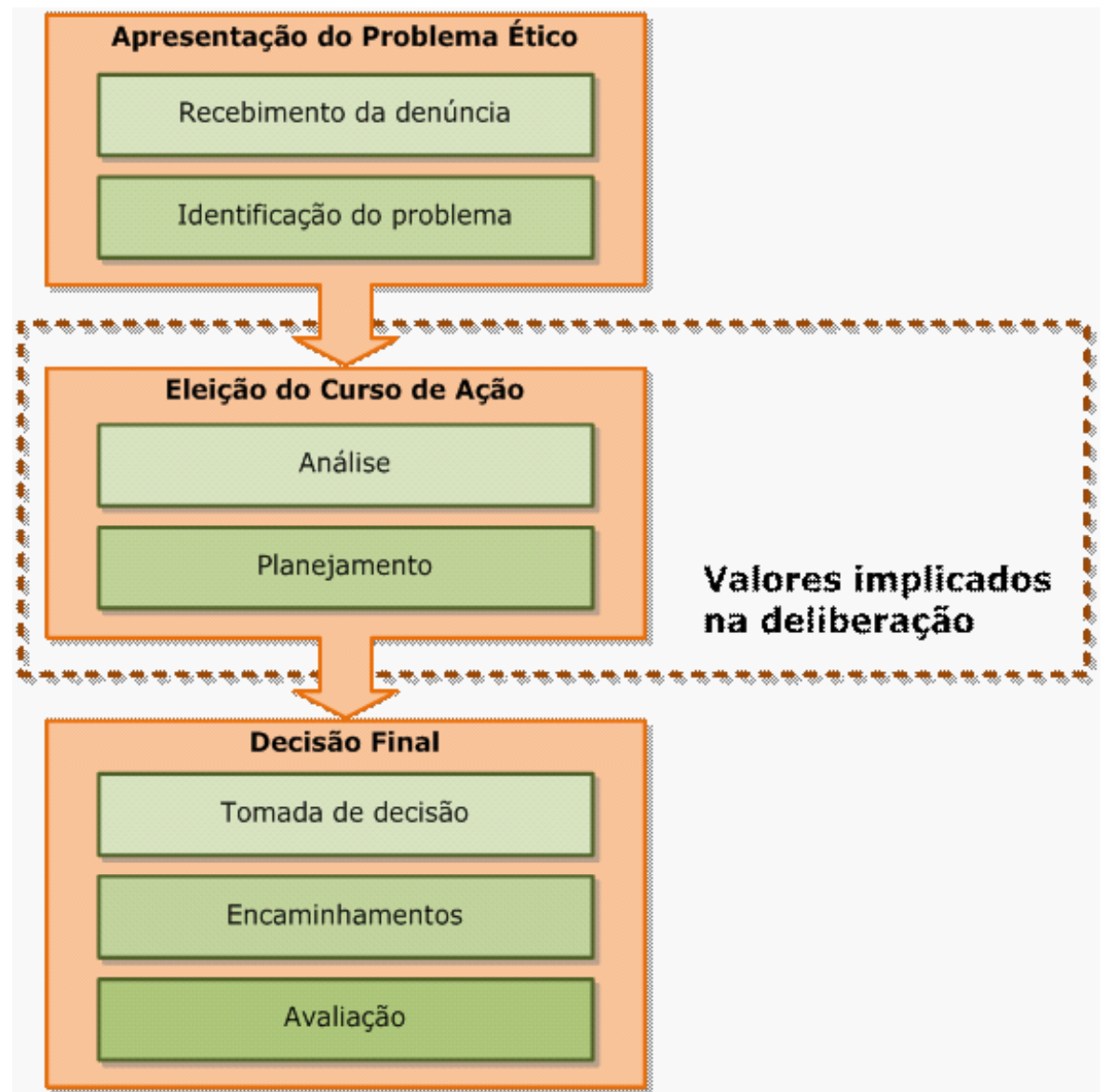

Figura 1. Etapas da deliberação dos problemas éticos vivenciados no processo de fiscalização dos profissionais de competência gerencial e fiscalizatória. Florianópolis, 2015. 
Na percepção dos profissionais de competência gerencial as etapas abrangem as seguintes atividades:

1을ecebimento da denúncia: Registrar a situação;

2을 Identificação do problema: Realizar a inspeção; Tomar ciência do fato; e Dialogar com os envolvidos;

3으 Análise: Avaliar o problema; Analisar o relatório de visita; Analisar os pontos positivos e negativos das ações a serem estabelecidas; Ponderar se a situação é nova ou reincidente; Buscar assessoria jurídica; Discutir o caso em equipe; e Fazer o diagnóstico da situação;

4ㅇ Planejamento: Estabelecer prioridades; Definir o plano de ação; e Socializar a definição dos planos de ação em equipe;

5으 Tomada de decisão: Promover ajustes entre os envolvidos; ou Encaminhar o processo para plenária; ou Encaminhar o processo para o presidente do Coren; ou Arquivar o caso se não procedente;

6을 Encaminhamentos: Delegar as atividades para os fiscais e acompanhar as ações estabelecidas; e Elaborar um relatório;

70 Avaliação: Avaliar todas as etapas do processo e refletir sobre o resultado final; se necessário, rever os planos de ação.

Já para os profissionais de competência fiscalizatória o itinerário deliberativo pode ser dividido em:

lo Recebimento da denúncia: Registrar a situação;

2ㅇIdentificação do problema: Realizar a inspeção; Dialogar com os envolvidos; Identificar os responsáveis; e Coletar o maior número de informações possíveis;

3ㅇ Análise: Estudar o problema ético; Avaliar a gravidade do caso; Conhecer as consequências da ocorrência; Ponderar os aspectos positivos e negativos; Analisar documentos; Discutir o caso em grupo (com chefia e colegas); Ponderar os riscos para os pacientes; e Fazer o diagnóstico da situação;

4ㅇ Planejamento: Propor soluções para o problema; Estudar as possibilidades de ação; Refletir sobre as consequências de cada opção; Estabelecer prioridades; Pensar nos prazos de acordo com o manual de fiscalização; Consultar assessoria jurídica; e Revisar todas as etapas para finalizar o processo;

5ㅇ Tomada de decisão: Orientar, ou Notificar, ou Abrir processo administrativo;

60 Encaminhamentos: Emitir parecer sobre a situação; Executar ações planejadas; Elaborar um relatório; e Enviar o processo para o setor responsável no Coren;

70 Avaliação: Acompanhar o processo; e, após o prazo estabelecido para a solução do problema, Retornar ao local para nova avaliação da situação.
A partir das etapas estabelecidas pelos participantes para deliberar sobre os problemas éticos vivenciados no cotidiano laboral, ampliou-se a discussão para identificar quais os valores envolvidos neste processo. Assim procedendo, tornaram-se evidentes alguns dos valores que amparam a realidade vivenciada, apresentadas no quadro 1.

Quadro 1. Valores que influenciam o processo de deliberação moral dos profissionais de competência gerencial e fiscalizatória. Florianópolis, 2015.

\begin{tabular}{|l|l|l|}
\hline \multicolumn{1}{|c|}{ Valores } & \multicolumn{1}{|c|}{ Gerentes } & \multicolumn{1}{c|}{ Fiscais } \\
\hline Éticos & Justiça. & $\begin{array}{l}\text { Imparcialidade, } \\
\text { Justiça, } \\
\text { Honestidade, } \\
\text { Responsabilidade, } \\
\text { Compromisso. }\end{array}$ \\
\hline $\begin{array}{l}\text { Normativos e } \\
\text { Deontológicos }\end{array}$ & $\begin{array}{l}\text { Leis, Normas, Código } \\
\text { de ética, Diretrizes } \\
\text { internas do Coren. }\end{array}$ & $\begin{array}{l}\text { Normas, Código de } \\
\text { ética, Legislação, } \\
\text { Resoluções, Manual } \\
\text { de fiscalização. }\end{array}$ \\
\hline Subjetivos & Razoabilidade. & $\begin{array}{l}\text { Bom senso, } \\
\text { Paciência, Amor } \\
\text { ao próximo, } \\
\text { Razoabilidade, } \\
\text { Altruismo. }\end{array}$ \\
& & $\begin{array}{l}\text { Conhecimento } \\
\text { teórico e Prático, } \\
\text { Orientações do } \\
\text { Cofen, Experiências } \\
\text { dos colegas, Diálogo } \\
\text { em grupo. }\end{array}$ \\
\hline Profissionais & $\begin{array}{l}\text { Conhecimento } \\
\text { teórico, Diálogo. }\end{array}$ & \\
& &
\end{tabular}

\section{DISCUSSÃO}

Etapas da deliberação moral na perspectiva dos profissionais de competência gerencial e fiscalizatória dos departamentos de fiscalização do exercicio profissional de enfermagem

Considerando uma visão macroscópica do processo de deliberação moral proposto, identificou-se entre ambos os grupos (gerentes e fiscais) as mesmas etapas de deliberação, apesar de perspectivas diferentes.

É importante destacar que o itinerário deliberativo proposto permitiu, por aproximação temática, estabelecer três grandes temas, que possibilitaram identificar os aspectos mais relevantes do processo: apresentação do problema ético; eleição do curso de ação; e decisão final.

A apresentação do problema ético implica na exposição da situação como um fato contraditório no âmbito dos valores e deveres. Este momento é de suma importância tendo em vista os desafios que envolvem o fenômeno com seus múltiplos sentidos éticos. Nesta perspectiva, julga-se imprescindivel ter consciência que os problemas não podem ser resolvidos com receitas prontas, mas com criatividade e guiado pelo conhecimento técnico-científico, por 
valores morais descritos no Código de Ética Profissional e na Lei do exercício profissional. A fim de conduzir a situação por um caminho prudente e responsável (2).

Ordenada através das atividades de recebimento da denúncia e identificação do problema, de modo geral, apresentou-se envolvida por trâmites organizacionais e burocráticos, ligados, certamente, a essência do trabalho destes profissionais no departamento de fiscalização, função gerencial e executora do processo(7).

Quanto à eleição do curso de ação consideraram-se duas atividades, uma envolvendo a análise e outra o planejamento. Para ambos os grupos a análise implica diretamente na operacionalização do processo de deliberação, sobretudo porque contribui para os cursos de ação. Identificou-se o empenho em analisar ao máximo a situação com base em alicerces sólidos como os elementos informativos, de apoio profissional, e discussão em grupo. Outro ponto significativo surgiu através da indicação, pelos fiscais, da ponderação dos riscos para os pacientes.

É sabido que a análise das circunstâncias e das consequências de um problema jamais será completa, sendo que alguns dados continuarão desconhecidos, porém deverá ser realizada de forma minuciosa, a fim de conduzir a situação por um caminho justo, prudente e realizável. Destacase que nesta etapa é de suma importância a imparcialidade, igualmente, pondera-se a legitimação de valores e regras relacionadas à profissão, a proteção aos vulneráveis e o respeito ao ser humano. Considera-se um momento de reflexão fundamentada e justificada que enriquece a compreensão do problema $a^{(1,8)}$.

Quanto ao planejamento ambos os grupos parecem atuar de maneira razoável e prudente, tentando, contudo, intervir de forma efetiva no problema. Entretanto, observou-se que os fiscais enfatizam aspectos diferentes dos gerentes, como a reflexão, o uso do manual de fiscalização e a reavaliação da situação. Sugere-se que o resultado está relacionado às experiências de cada grupo, ou ainda, às diferentes competências.

Por fim, a decisão final contempla as atividades de tomada de decisão, encaminhamentos e a avaliação. Nesta etapa, verificou-se um trabalho de ação complementar de ambos os grupos diante da decisão final do problema ético, reforçando o exercício de liderança dos gestores e o senso de responsabilidade dos fiscais. Observa-se ainda, que as etapas seguem um itinerário coerente capaz de guiar os profissionais efetivamente para a solução do problema.

De modo geral, deliberar é um meio sistematizado e contextualizado de refletir sobre os problemas éticos e/ou eventos da vida. O processo é complexo e envolve desde a subjetividade da situação, a falta de experiência na condução da problemática, até as dificuldades na etapa da tomada de decisão. $O$ método não se resume a certos requisitos formais, a ideia é ampliar os horizontes para contribuir com ações de respeito, promoção dos valores e da dignidade humana. Desta forma, sugere-se que os profissionais desenvolvam habilidades e competências deliberativas, independente da escolha do método, pois os problemas estão cada vez mais globais, no que tange os contextos socioculturais, políticos e institucionais ${ }^{(9,8)}$.

\section{Valores envolvidos no processo de deliberação moral}

Nesta perspectiva, os valores são fundamentos que norteiam os profissionais a tomarem decisões, assumindo um posicionamento seguro e legal no seu cotidiano laboral ${ }^{(10)}$.

Ao considerarmos os valores éticos, ambos os grupos enfatizaram a justiça, o que não se caracterizou como uma surpresa, já que a justiça pode ser considerada um valor constitutivo e essencial ao cargo. Temos que considerar ainda que na sociedade os indivíduos, de forma geral, são condicionados aos conceitos da moral supostamente reconhecidos em todo lugar ${ }^{(11)}$.

Outro aspecto importante é o enfoque legal a partir dos valores normativo e deontológico implicados no processo. Este resultado se deve, muito provavelmente, ao fato de que estes valores são a forma mais comum de orientação ética na prática profissional, além das peculiaridades de suas competências como agentes do Coren. Embora, não sejam identificados por todos os profissionais de enfermagem. Há que reconhecer-se a dificuldade da categoria de enfermagem em conhecer, compreender e aplicar as bases formais (Legislações, Código de Ética, Resoluções entre outros) no exercício profissional ${ }^{(10)}$.

A consulta ao manual de fiscalização, por parte dos fiscais, demonstra como este pode ser fonte de maior segurança diante dos problemas, sobretudo no que tange prazos regulamentares, providências para situações já previstas, além de condutas gerais a serem adotadas ${ }^{(7)}$.

O estudo permitiu revelar, ainda, o uso recorrente de valores subjetivos. A subjetividade é uma forma de expressão com variadas interpretações que envolvem o indivíduo e sua relação com o mundo ${ }^{(12)}$. Identificou-se como positivo a necessidade de outros valores na efetivação do itinerário deliberativo de ambos os grupos.

Por fim, quanto aos valores profissionais, percebe-se, de maneira global, que ambos os grupos apresentam uma bagagem ampla de conhecimentos. O que corrobora o fato de que os conhecimentos produzidos em nossa área são capazes de impactar no cotidiano laboral(13). 


\section{Limitações do estudo}

Como limitação do estudo destaca-se à indisponibilidade de estudos similares em enfermagem, o que por si só merece ser estudado a posteriori.

\section{Contribuições do estudo para a prática}

O presente estudo contribuiu para melhorar a compreensão acerca da importância e dos benefícios da fiscalização para a profissão e para a sociedade. Espera-se que as reflexões contidas nesta pesquisa promovam a valorização e um reconhecimento social do trabalho da enfermagem.

\section{CONCLUSÃO}

Ao revelar as etapas do itinerário deliberativo dos profissionais de competência gerencial e fiscalizatória, tornou-se possivel perceber que os trâmites do processo são fortemente vinculados a aspectos burocráticos e organizacionais, tornando evidente a hierarquia, a disciplina e a prioridade nas questões discutidas.

O itinerário proposto pelos participantes revela certa maturidade, constatada através das etapas eleitas, na medida em que se busca abordar o problema em toda sua complexidade, fundamentando as discussões a partir de valores, e possibilitando cursos de ações prudentes e razoáveis. Tais valores estão implicados em todo o processo e estão relacionados às dimensões ética, legal, subjetiva e profissional.

Em que pese que, independentemente do método utilizado podem ser produzidos resultados adequados, o conhecimento sobre bases teóricas e métodos pode ampliar a qualidade e o potencial crítico do processo de deliberação. É fundamental sensibilizar os profissionais, em diversos ambientes de trabalho para a prática de deliberação, promovendo a solução de problemas éticos de maneira mais dialógica e responsável. Para tanto, se faz necessário desenvolver habilidades e competências para contribuir com a discussão e com o rigor do processo.

\section{Contribuição dos autores}

Luciana Ramos Silveira e Flávia Regina Souza Ramos: concepção e/ou desenho; análise, interpretação dos dados e revisão final :Luciana Ramos Silveira, Flávia Regina Souza Ramos, Dulcinéia Ghizoni Schneider, María Isabel Saracibar Razquin e Laura Cavalcanti Farias Brehmer; redação Luciana Ramos Silveira; revisão crítica: Flávia Regina Souza Ramos.

\section{REFERÊNCIAS}

1. Gracia D. La deliberación moral: el método de la ética clínica. Revista Medicina Clinica [Internet]. 2001 [cited 2018 Jul 20]; 117(1):18-23. Available from: https://www.eutanasia.ws/hemeroteca/j62.pdf

2. Nora CRD, Zoboli ELCP, Vieira M. Problemas éticos vivenciados por enfermeiros na atenção primária à saúde: revisão integrativa da literatura. Revista Gaúcha de Enfermagem [Internet]. 2015 [cited 2018 Jul 20]: 36(1): 112-121. Available from: http://seer.ufrgs.br/index.php/ RevistaGauchadeEnfermagem/article/view/48809

3. Conselho Federal de Enfermagem (COFEN). Resolução COFEN no 374, de 23 de março de 2011. Normatiza o funcionamento do Sistema de Fiscalização do Exercicio profissional da Enfermagem e dá outras providências [Internet]. 2011 [cited 2018 Jul 20]. Available from: http://www. cofen.gov.br/wp-content/uploads/2012/03/resolucao374_anexo.pdf.

4. Caçador BS, Ramos FRS, Brito MJM. Processo de angústia/sofrimento moral em enfermeiros da estratégia saúde da família. Enferm Foco [Internet]. 2016 [cited 2018 Jul 20]; 7 (3/4): 22-26. Available from: http:// revista.cofen.gov.br/index.php/enfermagem/article/view/910/340

5. Machado MH, Santos MR, Oliveira E, Wermelinger M, Vieira M, Lemos W, Lacerda WF, Aguiar Filho W, Souza Junior PB, Justino E, Barbosa C. Condições de trabalho da enfermagem. Enferm Foco [Internet]. 2015 [cited 2018 Jul 20]: 6 (1/4): 79-90. Available from: http://revista.cofen.gov. br/index.php/enfermagem/article/view/695/305

6. Schneider DG, Ramos FRS. Moral deliberation and nursing ethics cases: Elements of a methodological proposal. Nursing Ethics [Internet]. 2012 [cited 2018 Jul 20]; 19(6): 764-776. Available from: http://journals. sagepub.com/doi/abs/10.1177/0969733011420096?journal Code=neja

7. Conselho Federal de Enfermagem (COFEN). Manual de Fiscalização do Cofen / Conselhos Regionais [Internet]. 2011 [cited 2018 Jul 20]
Available from: http://www.cofen.gov.br/wp-content/uploads/2012/03/ resolucao374_anexo.pdf.

8. Zoboli E. A aplicação da deliberação moral na pesquisa empírica em bioética. Revista Iberoamericana de Bioética [Internet]. 2016 [cited 2018 Jul 20]; 02: 01-19. Available from: http://revistas.upcomillas.es/index. php/bioetica-revista-iberoamericana/article/view/7348/7193

9. Gracia D. Tomar decisiones Morales: Del casuismo a la deliberación. Dilemata [Internet]. 2016 [cited 2018 Jul 20]; 8 (20): 15-31. Available from: https://dialnet.unirioja.es/servlet/articulo?codigo=5329386

10. Silva TN, Freire MEM, Vasconcelos MF, Silva Junior SV, Silva WJC, Araújo PS, Eloy AVA. Vivência deontológica da enfermagem: desvelando o código de ética profissional. Rev Bras Enferm [Internet]. 2018 [cited 2018 Jul 20]: 71(1): 7-15. Available from: http://www.scielo.br/pdf/reben/ v7ln1/pt_0034-7167-reben-71-01-0003.pdf

11. Corgozinho MM, Oliveira AASA. Equidade em saúde como marco ético da bioética. Saúde Soc [Internet]. 2016 [cited 2018 Jul 20]; 25(2): 431441. Available from: http://www.scielo.br/pdf/sausoc/v25n2/1984-0470 -sausoc-25-02-00431.pdf

12. Pivoto FL, Lunardi Filho WD, Lunardi VL, Silva PA. Organização do trabalho e a produção de subjetividade da enfermeira relacionada ao processo de enfermagem. Esc Anna Nery [Internet]. 2017 [cited 2018 Jul 20]; 21(1):e20170014. Available from: http://www.scielo.br/pdf/ean/ v21n1/1414-8145-ean-21-01-e20170014.pdf

13. Egry EY. Como o conhecimento pode impactar na qualificação da prática em enfermagem e saúde? Texto Contexto Enferm [Internet]. 2017 [cited $2018 \mathrm{Jul}$ 20]; 26(2): editorial. Available from: http://www.scielo.br/ pdf/tce/v26n2/pt_0104-0707-tce-26-02-editorial.pdf 